\title{
CRITICAL THINKING IN DESIGN
}

\author{
Marilyn DeLong \\ College of Design, University of Minnesota (USA)
}

\begin{abstract}
Trend thinking is about becoming aware of changes in the user within the context of a specific culture that can affect the future success of products in the marketplace and even the marketplace itself. Learning about abductive reasoning is important to understand for this type of critical thinking. This paper provides an overview of some concepts and processes used to promote critical thinking in an undergraduate upper level course taught in the College of Design at the University of Minnesota on communicating trends. Students who take the course come from the College of Design as well as various other colleges at the University of Minnesota.
\end{abstract}

Keywords: Design, trends, future, critical thinking, learning.

\section{Introduction}

This paper provides an overview of some concepts and processes used to promote critical thinking in an undergraduate upper level course taught in the College of Design at the University of Minnesota on communicating trends. Students who take the course are from the College of Design as well as various other colleges at the University of Minnesota and the course does not require design prerequisites. This means that students come from diverse disciplines, and concepts taught in the class cannot assume any former design experience.

Throughout the term, the class is organized into a team of 4-6 class members with each team composed of a spectrum of their self-defined expertise. Individuals within the team engage in a series of projects involving a user identified as other than oneself in the collective marketplace. Data must be collected and coordinated into a series of inferences by the team members to apply to a market segment - usually identified by age, gender and other characteristics, such as event, location including climate, season, as well as such characteristics as the preferences of the user. The schedule allows time for individuals and teams to present their work to the class for discussion and critique. Ample time is provided to allow the full class to digest how projects are organized: how to recognize individual and collective behaviors; how to optimize clues, symptoms, analogies, scenarios and hunches; and how to integrate concepts.

The concept of trends in the broadest sense, involves keeping abreast of those changes that occur within a culture and can be described as an anomaly or deviation from the norm (Raymond 2010). Trends can occur long term or short term. A trend can be century long and involve major life style changes. An example of a long-term trend is the $20^{\text {th }}$ century trend toward democratization of dress that involves casual dressing with fewer categories of clothing worn for a wide variety of occasions and that do not differentiate status. This has been possible with the increasing mass and global production that has resulted in affordable, albeit cheap products, often of low quality. Now everyone can afford this casual look! And with this long-term trend toward casual dressing has come short term trends accompanied by descriptive language. Terms such as athleisure, a combination denoting leisure and athletic wear, are coined to describe and market such democratizing trends related to specific products.

Trend thinking is critical thinking to learn how to observe the behavioral changes that are in process within a culture and how this might influence the user. Trend thinking activities thus demonstrate a process by which students can become acquainted with anticipating the future. Trends are a prediction of something that may likely occur in a certain way_-"specifically something that will be accepted by the average person" (Vejlgaard 2008, p.7). In this information rich era, what is "trending" is critical to understand for anyone involved in the design of consumer products or services. When design is approached from a human-centered, behavioral, change-making perspective, designer, maker, and user all become informants of the process and the associated trend analysis (Goncu-Berk 2018). 


\section{The process of trend thinking}

Trend thinking is reasoning that involves a combination of the familiar inductive, deductive reasoning and the less familiar abductive reasoning process (Fisher 2018). Abductive reasoning is sideways thinking in which the researcher engages in collecting data but then must consider how it all associates. It requires integrative skills, the result of which may not be considered entirely logical. It often takes a creative leap to come forward with something new and useful. Abductive thinking proceeds in an orderly and methodical way toward the development of useful inferences. Inferences result from hunches, clues, symptoms, analogies, and scenarios. To accomplish this kind of critical thinking requires intensive data collecting and the application of abductive reasoning.

When compared with the process of design thinking, the process of trend thinking has some similarities. Aspelund (2015) outlines steps or stages of the design process. He explains that at every stage, the student must pause, evaluate, and reflect upon the research being done. In like manner the process of trend thinking involves a step-by-step process based upon following a procedure, and evaluating and reflecting upon the outcome. Design thinking according to Kelly and Littman (2001) is research involving the user in defining the problem.

The process of trend thinking means learning through a series of experiences that provide building blocks that inform outcome. Research begins with techniques for learning how to examine the pertinent data through both desk and field research. Desk research delves into literature on theories about the direction of trends, who is involved and at what pace. For example, the user is characterized in terms of who is first and last to follow a trend direction, length of time a trend exists according to certain characteristics. Field research involves how to observe and take note of what is happening around you, being curious, flexible, and open to change. It means being willing to characterize first you as the user in terms of likes and dislikes, life-style preferences, attitudes and behavior and then to discern this experience of individual characterization in others. As the researcher collects data, the relationship of the forecast to the user, the product and marketplace become input for the trend. The following exercise, "From me to we" helps a student to understand himself as the user of products and how that translates to self as part of a collective.

\subsection{From me-to-we}

Because apparel design, production and marketing usually involve the concept of the collective, this product category is useful for trend thinking as an educational tool that allows the student researcher to learn the difference in perspective of oneself as an individual compared to another, and then how the perspective of oneself informs collective behavior (Blumer 1969). Change in the expression of self involves both individual and collective behavior. An example of individual expression would be if a person strives to be uniquely individual in dressing, eating, or in choices of entertainment and stands out in this individual expression from peers; such a leader in the trend is often characterized as an early adopter. An example of collective expression would be if a person notices that those in the cohort group of the trend leader are thinking or acting in a similar way. There is a tension created when a person wants to conform as both part of a collective expression and at the same time, wants to express himself individually.

From "me-to-we" is a useful concept in trend thinking, First the researcher must examine and understand his or her own behavior -i.e. needs, desires, likes and dislikes. Through self-directed questions, this exercise probes favored product attributes. The student researcher can become aware of his own preferences and by comparing them to others on his team, can sort out similarities to others within his cohort group. An objective in this process is to eventually move outside one's own cohort group and learn how to discover the behaviors of another external group different from one's familiar cohort in such aspects as age, preferences and lifestyle. Learning the perspective of another group is useful when designing or marketing products.

\subsection{From visual to verbal}

Documenting is an important means of collecting data that can provide both visual and verbal insights. Because students in this class are not always used to the power of the visual and relating the visual to verbal explanations, they are encouraged to collect such data in a notebook. Most students have access to a smart phone and both the camera and audio recording features included can be used to document both desk and field research. Students learn the importance of the visual as well as how to use the visual optimally to provide verbal detail and richness to their findings. Specific meanings can come from analysis of data derived from photo documentation. Students are encouraged to engage in exploring the visual as means to develop or interpret the verbal. Such notebooks vary in their use, but as students become familiar with the opportunities of this assignment, they often explore visual relationships through 
collage, for example placing photos along a timeline or on a continuum according to positive or negative reactions of the user.

Once the student researcher learns about these two concepts, e.g. me-to-we and navigating visual and verbal connectional information, then one can move on to the user being studied as an individual and as a collective. Now the student proceeds to desk and field research.

\section{Desk research}

\subsection{Key quotes}

An example of desk research involves integration through significant concepts addressed in the literature. Students are asked to integrate concepts when examining data that already exist, such as census data or readings that address existing trend theories, and ideas of forecasters that can be applied to their projects. To do this, students are asked to select a "key quote" from a current reading assignment and then to find support or an expansion of that idea from previous readings. Writing a summary coordinating ideas from the readings is the final task. This integration of readings requires reflection and abductive reasoning. Desk research of a student who integrated readings from several sources follows:

"This quote relates heavily to trend thinking because forecasting is at the center of trend identification and analysis. This article lays out the types of forecasting and necessary sources of information needed to make accurate forecasts about fashion trends. Trend thinking requires one to think as a forecaster by analyzing the greater global markets across multiple industries to identify mega and mini trends that can affect certain consumer segments. Forecasting does just that through specialized professions with specialized education and experiences. Mega trends are those that reflect a cultural shift that becomes influential in changing the way people view themselves. An example of a mega-trend is the greater acceptance of gender neutral and non-binary clothing. Mini-trends are those that are fleeting and evolve from season to season. This could be a color, fabric or silhouette that is gone by next season (DeLong, 2018)."

(Report of student in class 3217.)

This assignment repeated throughout the semester forces the student to reflect upon and integrate readings. Scenarios can further critical thinking.

\subsection{Scenarios}

Futurists who often create more expansive scenarios provide a useful basis for predicting adjacent trends, such as the increase in the use of Internet retail and e-commerce, or social networking (Singh 2012). Most industry professionals spend time thinking about how forecasts will affect the success of their products within the available markets, and scenarios help to focus and enhance the exercise of integrating readings for students. A scenario narrative explores the future use of a product from a user's lifestyle point of view. Such scenarios can make design ideas more explicit. As Martin and Hanington (2012) suggest, scenarios should focus more on what technology might enable than on the details of technology. For example, the above student quote from the readings could be explored further with the following scenario:

Future Scenario: In year 2035 the number of retail stores has diminished, and retail has gradually given way to online shopping for a majority of users in the US. In addition, the government has urged all populations to adopt sustainable products because of the sheer glut of non-biodegradable products - both new and used-- that are stockpiled around the country.

Questions: How would the design of products change under these circumstances? How could individual expression be achieved? What could become a mega-trend?

Solutions considered by students include products designed for second use; products that are biodegradable; multiples designed in basic shapes and colors to serve both the gendered and gender-neutral markets; body modifications such as tattoos to achieve individual expression.

The teams address outcomes of such a scenario: the differences that could occur in changes in shopping behavior; design for those seeking gender neutral clothing; how designers would change the product.

Such scenarios call for further integration of ideas that are best informed when backed by research. The teams who engage in such a strategic planning tool help create a shared product vision for the future. But such a shared vision involves field research. 


\section{Field research}

\subsection{Participant observation}

In sociology and anthropology participant observation is a process by which one learns to maintain a sense of objectivity while participating in a familiar or unfamiliar activity -The process involves first being a careful observer, a good listener and being open to the unexpected (Kawulich 2005). Second, this process means once you have walked away from the activity being observed, to quickly record what is remembered and then to distance yourself from the immediate activity to analyze what is happening. At first the activity can be a familiar one. Students have selected; riding on the bus between campuses, eating out at a restaurant, attending a theatrical production, or handing out treats at Halloween in their neighborhood. For example, here is a student observing and reflecting upon how current experience compares with her past experience of dressing in costume for Halloween:

"For this event, I observed the trick or treating event at $50^{\text {th }}$ and France storefronts on Saturday, October 27. Growing up, the majority of costumes I would see were store bought and consisted of a lot of superhero's, princesses, and popular TV or movie characters. During the trick or treating event I barely saw any boys dressed as a super hero or a girl decked out in a princess dress from the Disney store. The majority of the costumes were either DIY ideas found on Pinterest or food, which was very surprising. Children were dressed as Tacos, donuts and watermelon which made me laugh because that is something I never would have thought to dress up as a child. The most creative DIY costume I saw was a cup of hot coco. The girl had made her costume as a mug and put little white balloons around her neck area to act as marshmallows. She had even added a design to the fabric mug and monogrammed her initials on it. I really appreciated the out-of-the-box thinking by both parents and their kids when it came to selection of a Halloween costume to wear this year. It shows that as consumers become more creative, they are less willing to pay for the over-priced, low quality, generic costumes that can be purchased ready-made." (Report of Student in class 3217)

This means being flexible with what is observed and being able to relate observation to past experience. With practice in participant observation, one can move from the familiar to observe the unfamiliar. It takes effort to go beyond the familiarity of one's own experience and group to consider another group without becoming resistant and judgmental in the outcome. It means learning about being an astute observer and learning to create a certain distance not only from your familiar group but also to the other group.

\subsection{Make the familiar-strange}

Learning how to make the familiar strange is a possible outcome of participant observation. It means bringing to awareness the habits that influence us. Only when we do not take for granted those familiar experiences can we accept some measure of objectivity in our thinking. Then the "what if" and the "where for" can begin to take shape. Here is a student quote that begins to address the "what if":

"Sustainability has been a crucial factor in many parts of society that has hit a high in recent years, especially in fashion. Many fashion brands have recognized the importance of the environment and sustainability and have made significant changes to how they operate and manufacture clothing. However, many of these companies still operate in the realm of fast fashion. Due to this, consumption increases and will remain high even with their green initiatives to combat environmental issues, because consumers will continue to buy goods and not stop." Report of Student in class 3217

This significant quote could be expanded with a scenario or at least a discussion of fast fashion and the current concept of slowing the pace of fashion and what sustainable practices could be involved (Clark 2008).

\section{Conclusion}

These concepts and processes are examples of trend thinking that require much reflection that is not only individual but also involves group thinking. The important trend thinking occurs when the user is considered within the context of the current time but also becomes part of the prediction for the future. As students become informants in the trend thinking process, they can regard change to be related to our physical and cognitive needs, and to our social and emotional needs. If they consider change in this way, they could trend toward having a more sustainable world because the design and marketing of products would be based upon abductive reasoning that takes serious data collection using both desk and field research and provides an opportunity to engage in this type of reasoning process that relies on hunches, inferences and "what ifs". 


\section{References}

Aspelund, K. $3^{\text {rd }}$ Edition. (2015). The Design Process. New York: Bloomsbury.

Blumer H. (1969). Fashion: From Class Differentiation to Collective Selection. The Sociological Quarterly 10: 3. pp. 275-291.

Clark, H. 2008. Slow + Fashion - an Oxymoron-or a Promise for the Future? Fashion Theory, 12(4), pp. 427-446.

DeLong, M. (2018). Developing an Expert's Viewpoint," Communicating Fashion: Trend Research and Forecasting. http://open.lib.umn.edu/communicatingfashion/.

Fisher, T. (2016). Designing our Way to a Better World. Minneapolis: University of Minnesota Press.

Goncu-Berk, G. (2018). The Trend Research Toolkit. Communicating Fashion: Trend Research and Forecasting. http://open.lib.umn.edu/communicatingfashion/.

Kelly, T. \& Littman, J. (2001). The Art of Innovation: Lessons in Creativity from IDEO, America's Leading Design Firm. New York: Doubleday.

Martin, B. \& Hanington, B. (2012). Universal Methods of Design. Beverly, MA: Rockport.

Raymond, M. (2010). The Trend Forecaster's Handbook. London: Laurence King.

Singh, S. (2012). New Mega Trends, Implications for our Future Lives. New York: Palgrave Macmillan.

Vejlgaard, H. (2008). Anatomy of a Trend. New York, NY: McGraw-Hill 